\title{
Genome-wide identification and expression profiling of the fatty acid desaturase gene family in the silkworm, Bombyx mori
}

\author{
Q.M. Chen, D.J. Cheng, S.P. Liu, Z.G. Ma, X. Tan and P. Zhao \\ State Key Laboratory of Silkworm Genome Biology, Southwest University, \\ Chongqing, China \\ Corresponding author: P. Zhao \\ E-mail: zhaop@swu.edu.cn
}

Genet. Mol. Res. 13 (2): 3747-3760 (2014)

Received August 21, 2013

Accepted December 6, 2013

Published May 13, 2014

DOI http://dx.doi.org/10.4238/2014.May.13.2

\begin{abstract}
Fatty acid desaturases exist in all living organisms and play important roles in many different biologic processes, such as fatty acid metabolism, lipid biosynthetic processes, and pheromone biosynthetic processes. Using the available silkworm genome sequence, we identified 14 candidate fatty acid desaturase genes. Eleven genes contain 3 conserved histidine cluster motifs and 4 transmembrane domains, but their N-terminal residues exhibit obvious diversity. Phylogenetic analysis revealed that there are 6 groups; Bmdesat 1 and Bmdesat5-8 were clustered into group 2, which is involved in $\Delta 11$ desaturation activity, and Bmdesat3-4 were grouped in group 1, which is involved in $\Delta 9$ desaturation activity. Twelve of the 14 genes have expressed sequence tag evidence. Microarray data and reverse transcription polymerase chain reaction analysis demonstrated that Bmdesat3-4 and Bmdesat10 were expressed from the larval to moth stages and in multiple tissues on day 3 of 5th instar larvae. Bmdesat9, Bmdesat11, and Bmdesat14 were expressed during the pupal and late-embryonic stage, suggesting that they may take part in fatty acid metabolism to provide energy. These results provide some insights into
\end{abstract}


the functions of individual fatty acid desaturases in silkworm.

Key words: Reverse transcription polymerase chain reaction; Bombyx mori; Fatty acid desaturase genes; Multiple sequence alignment; Expression profiles

\section{INTRODUCTION}

Fatty acid desaturases exist in bacteria, fungi, plants, and animals, and they can convert a $\mathrm{C}-\mathrm{C}$ single bond to a $\mathrm{C}=\mathrm{C}$ double bond at specific positions in a fatty acyl chain. They are classified into 3 categories according to diverse substrates, namely acyl-CoA, acyl-lipid, and acyl-acyl carrier protein (ACP) desaturases (Los and Murata, 1998). Acyl-CoA desaturases are present in animals, yeast, and fungal cells; acyl-lipid desaturases are in plants and/or cyanobacteria; and acyl-ACP desaturases are in the plastids of plant cells. Acyl-CoA and acyllipid desaturases are hydrophobic proteins with 4 transmembrane domains, whereas acyl-ACP desaturases are soluble proteins (Shanklin et al., 1994; Murata and Wada, 1995).

Fatty acid desaturase was first characterized from rat and consists of 358 amino acid residues (Thiede et al., 1986). Recently, fatty acid desaturases have been broadly analyzed in many species. Acyl-CoA desaturase genes from vertebrates, including Siganus canaliculatus (Li et al., 2010), Danio rerio (Hastings et al., 2001) and Homo sapiens (Li et al., 1994) have been cloned. In insects, 3 and 2 acyl-CoA desaturases were identified in Drosophila melanogaster (Wicker-Thomas et al., 1997; Dallerac et al., 2000; Chertemps et al., 2006) and Tribolium castaneum (Horne et al., 2010), respectively. In moths, many fatty acid desaturase genes were cloned from pheromone glands, including $\Delta 11$ and/or $\Delta 9$ desaturase in Trichoplusia $n i$ (Knipple et al., 1998), Helicoverpa zea (Rosenfield et al., 2001), Bombyx mori (Yoshiga et al., 2000; Moto et al., 2004), Helicoverpa assulta (Jeong et al., 2003), Manduca sexta (Matouskova et al., 2007), and Ostrinia spp (Roelofs et al., 2002); $\Delta 14$ desaturase in Ostrinia spp (Roelofs et al., 2002); and $\Delta 10$ desaturase in Planotortrix octo (Hao et al., 2002).

Fatty acid desaturases of insects are structurally and biochemically homologous to the $\Delta 9$ acyl-CoA desaturases from fungi and animals, suggesting that they originate from a common ancestor (Stukey et al., 1990; Shanklin and Cahoon, 1998). They are a class of integral proteins residing in the endoplasmic reticulum that contains non-heme iron atoms at their active site (Strittmatter et al., 1974), and they function as component of a multienzyme complex consisting of $\Delta 9$ acyl-CoA desaturases, NADH-cytochrome $\mathrm{b}_{5}$ reductase (a flavoprotein) and cytochrome $b_{5}$ (a hemoprotein) (Holloway, 1971; Spatz and Strittmatter, 1971; Rogers and Strittmatter, 1973). In most insects, fatty acid desaturases that are involved in sex pheromone biosynthetic pathways were widely studied; however, there is little research on the fatty acid metabolism, regulation of cell membrane fluidity to adapt to temperature fluctuations, and production of beneficial materials (such as pupal oil). Further studies will help us comprehensively understand the multiple functions of insect fatty acid desaturases.

The silkworm, B. mori, is an important economical insect and a model organism for Lepidoptera (Goldsmith et al., 2005). To date, only 4 desaturase genes were reported; one of them, originally named desat1, possesses both Z11 desaturation and $\Delta 10,12$ desaturation activities, which are responsible for the 2 desaturation steps in the biosynthesis pathway of bombykol, (E,Z)-10,12-hexadecadien-1-ol (Moto et al., 2004). The functions of other desaturases are still not well known, and in the past decades, the main focus was on the molecular mechanisms underly- 
ing sex pheromone production in the silkworm (Matsumoto et al., 2007; Matsumoto, 2010). Thus, in this study, we first identified the fatty acid desaturase genes based on the complete silkworm genome sequence; then, multiple sequence alignment of silkworm desaturases and phylogenetic analysis were performed, and the spatio-temporal expression of these genes was also investigated via microarray data and reverse transcription polymerase chain reaction (RT-PCR) analysis.

\section{MATERIAL AND METHODS}

\section{Identification of fatty acid desaturase genes in silkworm and other insects}

Five desaturase sequences from silkworm were downloaded from GenBank and were used as queries against the silkworm database using basic local alignment search tool (BLAST), with an E-value threshold of $10^{-6}$. Predicted sequences were validated by aligning them to the non-redundant dataset and the expressed sequence tag (EST) database (threshold E value $<10^{-30}$, identities $>90 \%$, and match lengths $>100 \mathrm{bp}$ ). All of the identified fatty acid desaturases were further checked for the 3 conserved histidine tracks, which were used to characterize the fatty acid desaturases (Los and Murata, 1998). The protein sequences of Drosophila melanogaster, Anopheles gambiae, Apis mellifera, and Tribolium castaneum were downloaded from the National Center for Biotechnology Information (NCBI). We used fatty acid desaturases from silkworm as queries in a homology search for the 4 insects fatty acid desaturases.

The chromosome distribution of fatty acid desaturase genes in silkworm and the 4 other surveyed insects was analyzed by SilkMap (http://silkworm.swu.edu.cn/silksoft/silkmap. html) and the Map View tool on http://www.ncbi.nlm.nih.gov/projects/mapview/ and http:// genome.ucsc.edu/, respectively.

\section{Prediction of silkworm fatty acid desaturase characteristics}

Predictions of a transmembrane helix of silkworm fatty acid desaturases were performed by TMHMM2.0 (http://www.cbs.dtu.dk/services/TMHMM-2.0/) and TMpred (http:// www.ch.embnet.org/software/TMPRED_form.html). Endoplasmic retention signals were predicted by PSORT (http://psort.hgc.jp/form2.html). The protein domains were predicted by InterProScan (http://www.ebi.ac.uk/Tools/pfa/iprscan/).

\section{Phylogenetic analysis}

Amino acid sequences of fatty acid desaturases were aligned using 2 methods, Clustal $\mathrm{X} v 1.83$ and Muscle v3.6. Using a conserved core domain, we reconstructed the phylogenetic tree by the neighbor-joining (NJ) methods in the program MEGA5. The evolutionary distance was assessed by the JTT amino acid matrix using complete deletion option, and the tree was tested by bootstrapping with 1000 resampling replicates.

\section{Sample preparation}

We collected individuals of the silkworm strain Dazao at 27 different time points from day 1 to day 10 after oviposition (E1-E10) and from day 3 of the 5th instar stage (V3) to moths, including V3, V5, V7, and W12 (12 h after wandering); W24, W36, and W48 (completion 
of spinning); W60 (pupation); W72, W96, and W120 (formation of egg); and W144, W168, W192, W216, W240, and adult (moths) for both sexes; all samples were immediately frozen with liquid nitrogen and stored at $-80^{\circ} \mathrm{C}$ until use.

\section{Expression profiling of silkworm fatty acid desaturase genes}

Total RNA was extracted using Trizol reagent (Invitrogen, USA), which was followed by treatment with RNase-free DNaseI (Promega, USA) for $30 \mathrm{~min}$ at $37^{\circ} \mathrm{C}$ to eliminate the contaminating genomic DNA. The first strand of cDNA was synthesized using M-MLV reverse transcriptase (Invitrogen, USA) for $1 \mathrm{~h}$ at $42^{\circ} \mathrm{C}$. All cDNA samples were normalized using the silkworm cytoplasmic actin A3 gene as an internal control (forward primer: 5'-AACACCCCGTCCTGCTCACTG-3'; reverse primer: 5'-GGGCGAGACGTGTGATTTCCT-3'). All gene-specific primers for semi-quantitative RTPCR detection are listed in Table S1. PCR amplification was performed in a total reaction volume of $25 \mu \mathrm{L}$ containing normalized cDNA, 10 pmol each primer, $2 \mathrm{mM} \mathrm{MgCl}, 0.25$ $\mathrm{mM}$ dNTP, $1 \mathrm{X}$ buffer, and 2.5 units Taq DNA polymerase. RT-PCR were performed using the following program: initial incubation at $94^{\circ} \mathrm{C}$ for $4 \mathrm{~min} ; 25$ cycles at $94^{\circ} \mathrm{C}$ for $40 \mathrm{~s}, 40 \mathrm{~s}$ of annealing (temperatures listed in Table S1), and $40 \mathrm{~s}$ to $1 \mathrm{~min}$ extension at $72^{\circ} \mathrm{C}$ (the time depends on the length of the gene); and final extension at $72^{\circ} \mathrm{C}$ for $10 \mathrm{~min}$. PCR products were separated on $1.5 \%$ agarose gels and stained with ethidium bromide. We also analyzed the expression patterns of silkworm fatty acid desaturase genes from microarray data that were described in previous studies (Xia et al., 2007). Microarray-based gene expression was visualized using GeneCluster 2.0.

\section{RESULTS}

\section{Identification of fatty acid desaturase genes in silkworm and other insects}

We used the sequences of previously reported fatty acid desaturases as queries to search against the silkworm genome sequence by BLAST and identified a total of 14 putative fatty acid desaturase genes (Table 1). Bmdesat1 and Bmdesat2 (GenBank accession No. AF182405) shared 98\% identity in their proteins; therefore, they were considered to be alleles from hybrid strains (Yoshiga et al., 2000; Moto et al., 2004). Bmdesat13-15 lacked the N-terminal region containing the first histidine box; this may be because of gaps in the silkworm genome or pseudogenes.

Bmdesat5 and Bmdesat13 are located on nscaf2959 and scaffold 798, respectively; they could not be mapped to chromosomes in the current version of the silkworm genome sequence. The other 12 genes reside on 7 chromosomes in a nonhomogeneous manner. Among them, there are 5 genes on chromosome 12, 2 genes on chromosome 24, and only 1 gene on each of the other chromosomes. Bmdesat10, Bmdesat12, and Bmdesat15 are tandemly arranged in the middle region of chromosome 12, whereas Bmdesat 4 is separately located on the same chromosome apart from the tandem set (Figure S1).

The inventories of fatty acid desaturase genes in the other 4 insects were also identified, including D. melanogaster, A. gambiae, A. mellifera, and T. castaneum. There are 7 candidate fatty acid desaturase genes in D. melanogaster, 6 in A. gambiae, 5 in Apis mellifera, and 15 in T. castaneum (Table 2). All of the fatty acid desaturase genes in D. melanogaster and A. gambiae are distributed on chromosome 3 and chromosome 2R, respectively. Most of 
the fatty acid desaturase genes in T. castaneum are located on group 6, whereas only 1 fatty acid desaturase gene in A. mellifera could be mapped to LG12 based on the current genome assembly (Table 2 and Figure S1). The number of introns in fatty acid desaturase genes varied from 0 to 6 in the 5 insect species. Only D. melanogaster desatF (CG7923), which originated from a single retrotransposition event, has no intron (Bai et al., 2007).

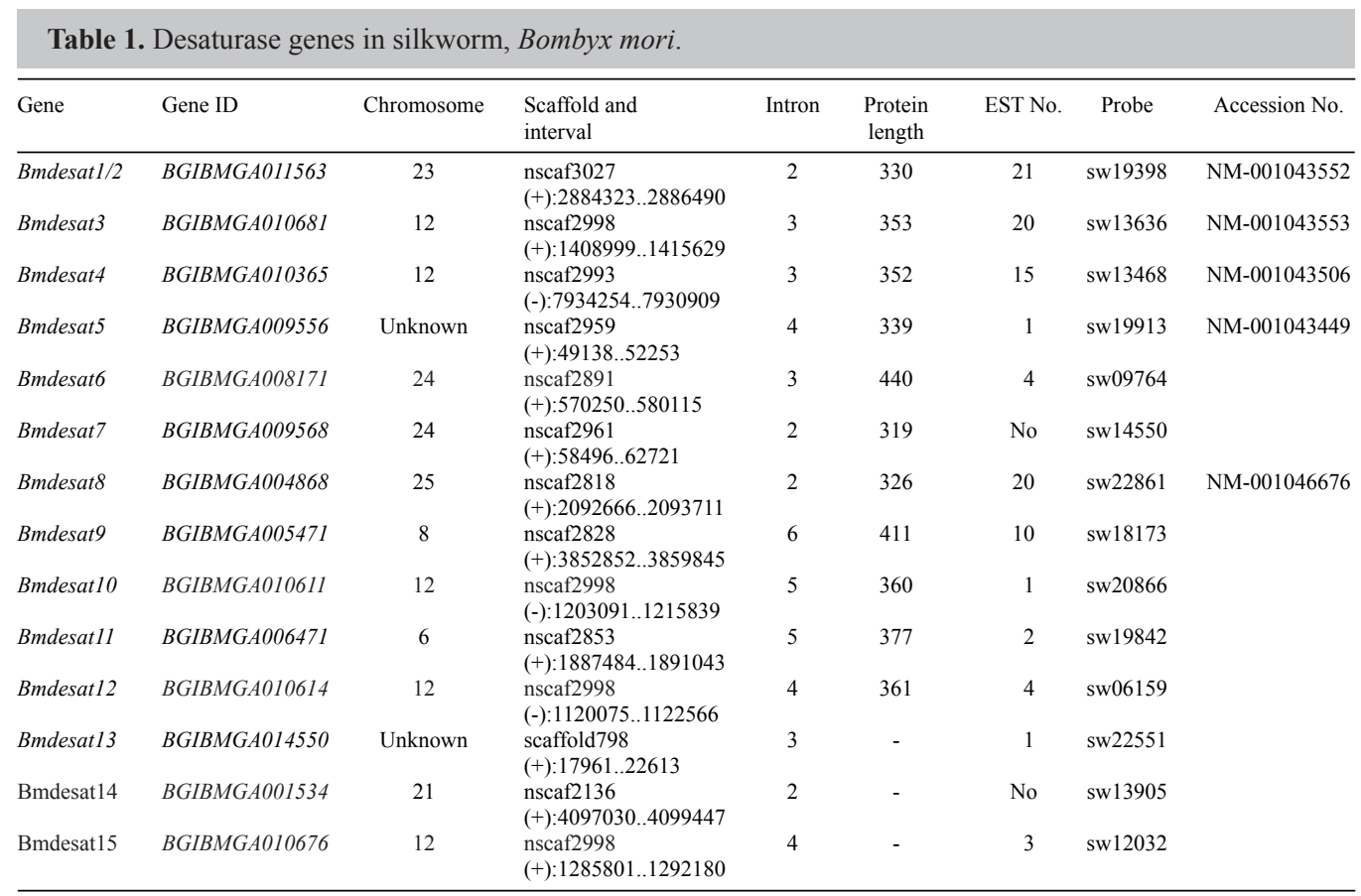

(-) The corresponding desaturase gene may be incomplete.

\section{Characteristics of the silkworm fatty acid desaturases}

Multiple sequence alignment of silkworm fatty acid desaturases showed a substantial conservation in the region of the fatty acid desaturase core domains (delimited by GAHR and EGFH motifs) (Figure 1). In contrast, great diversity was noted in sequence length and order at the N-terminus of silkworm fatty acid desaturases. Three histidine clusters (labeled $\mathrm{H} 1, \mathrm{H} 2$, and H3) are common features of fatty acid desaturases and might be essential for desaturase catalytic activity (Shanklin et al., 1994). All silkworm fatty acid desaturases with complete sequences have 4 transmembrane domains, which are marked TM1-TM4. Eight silkworm fatty acid desaturases comprise the proposed endoplasmic retention signal.

It is obvious that the fatty acid desaturases of silkworm display distinct signature motifs based on a group of 4 amino acids that are upstream of $\mathrm{H} 3$. The signature motifs of Bmdesat1, Bmdesat5, Bmdesat6, and Bmdesat7 are xxxQ; that of Bmdesat3 is NPVE; and that of Bmdesat4 is KPSE. The amino acid sequences of fatty acid desaturases containing the xxxQ signature motif encode $\Delta 11$ desaturases, and the sequences containing the NPVE and KPSE signature motifs encode $\Delta 9$ desaturases (Knipple et al., 2002). 
Table 2. Summary of fatty acid desaturase genes in other four insects.

\begin{tabular}{|c|c|c|c|c|}
\hline Gene name gene ID & Accession No. & Chromosome location & Intron & Protein length \\
\hline \multicolumn{5}{|l|}{ Drosophila melanogaster } \\
\hline Desat1 CG5887 & NM_169468 & $3 \mathrm{R}(+): 8270531 . .8272660$ & 3 & 383 \\
\hline Desat2 CG5925 & NM_141944 & $3 \mathrm{R}(+): 8262143 . .8263478$ & 3 & 361 \\
\hline CG8630 & NM_169500 & $3 R(+): 9108127 . .9109672$ & 3 & 408 \\
\hline Fad2 CG7923 & NM_143709 & $3 \mathrm{~L}(+): 11016639 . .11017703$ & 0 & 355 \\
\hline CG9747 & NM_143522 & $3 R(-): 26011569 . .26016463$ & 3 & 461 \\
\hline CG15531 & $\mathrm{NM}^{-} 143523$ & $3 R(-): 26020608 . .26021890$ & 3 & 334 \\
\hline CG9743 & $\mathrm{NM}^{-} 143524$ & $3 R(-): 26022362 . .26024615$ & 4 & 420 \\
\hline \multicolumn{5}{|l|}{ Anopheles gambiae } \\
\hline AGAP001713 & XM_321375 & $2 \mathrm{R}(+): 8873957 . .8875611$ & 3 & 355 \\
\hline AGAP003049 & XM_001237392 & $2 \mathrm{R}(-): 31439418 . .31452934$ & 4 & 405 \\
\hline AGAP003050 & XM_311821 & $2 R(-): 31465070 . .31466450$ & 3 & 363 \\
\hline AGAP003051 & XM_311819 & $2 \mathrm{R}(-): 31474690 . .31476162$ & 3 & 381 \\
\hline AGAP003418 & XM_311704 & $2 \mathrm{R}(+): 39199869 . .39204460$ & 3 & 394 \\
\hline AGAP004572 & $\mathrm{XM}^{-} 313877$ & $2 \mathrm{R}(+): 59481796 . .59490161$ & 5 & 415 \\
\hline \multicolumn{5}{|l|}{ Apis mellifera } \\
\hline GB11969 & XM_395629 & Unknown & 4 & 347 \\
\hline GB11596 & XM_623922 & Unknown & 5 & 368 \\
\hline GB15513 & XM-624791 & Unknown & 4 & 291 \\
\hline GB17206 & $\mathrm{XM}-624554$ & Unknown & 4 & 368 \\
\hline GB18070 & - & LG12(-):3184837..3186686 & 4 & 336 \\
\hline \multicolumn{5}{|l|}{ Tribolium castaneum } \\
\hline GLEAN 00549 & XM_968940 & LG2(-):852711..851338 & 4 & 335 \\
\hline GLEAN_03656 & NM_001143734 & LG3(+):188726..189951 & 3 & 358 \\
\hline GLEAN_15108 & EFA05022 & LG6(-):54316..50512 & 3 & 323 \\
\hline GLEAN_15349 & XM_963735 & LG6(-):116035..115397 & 4 & 321 \\
\hline GLEAN_15382 & XM_963804 & LG6(+):116215..117271 & 2 & 318 \\
\hline GLEAN_15383 & XM_963877 & LG6(+):117625..118971 & 2 & 320 \\
\hline GLEAN_15338 & XM-965427 & LG6(-):242416..241459 & 2 & 290 \\
\hline GLEAN_15395 & EFA05245 & LG6(+):263878..264992 & 2 & 329 \\
\hline Z9desA GLEAN_11471 & NM_001195235 & LG10(+):607006..608123 & 1 & 353 \\
\hline GLEAN_16399 & EFA 12397 & Unknown & 3 & 277 \\
\hline Z9desB GLEAN_16415 & NM_001193649 & Unknown & 1 & 350 \\
\hline GLEAN 14821 & EFA 12799 & Unknown & 3 & 389 \\
\hline GLEAN 14820 & EFA12798 & Unknown & 5 & 414 \\
\hline GLEAN_14819 & XM_962850 & Unknown & 4 & 356 \\
\hline GLEAN 16414 & EFA 12412 & Unknown & 4 & 288 \\
\hline
\end{tabular}

\section{Phylogeny of the insect fatty acid desaturase family}

According to multiple sequence alignments of the conserved core region of insect fatty acid desaturases (Figure S2), we constructed the NJ tree using the program MEGA5.02 (Figure 2). The phylogeny showed that the deep internal branches were very short, suggesting that the duplication events causing the insect fatty acid desaturase gene families occurred within a narrow period. The phylogeny also showed that the internal branches within different clusters were very short but the terminal branches were much longer, suggesting that the changes that correlated with speciation occurred quickly. The tree could be divided into 6 groups (group 1-6). Group 1 had 2 subgroups, $\Delta 9(16 \mathrm{C}>18 \mathrm{C})$ and $\Delta 9$ (18C $>16 \mathrm{C})$ lineages, based on the catalytic activity of previously reported fatty acid desaturases. Fatty acid desaturases containing the $\mathrm{xxxQ}$ signature motif were in group 2; these were identified only from Lepidoptera, suggesting that the group may be Lepidoptera-specific. Other desaturases that clustered into groups 3-6 were distantly related to the functionally known fatty acid desaturases in group 1 and group 2, suggesting that they are likely to evolve new functions by an independent route, such as the $\Delta 14$ desaturases in Ostrinia spp (Roelofs et al., 2002) and $\Delta 12$ desaturase of 


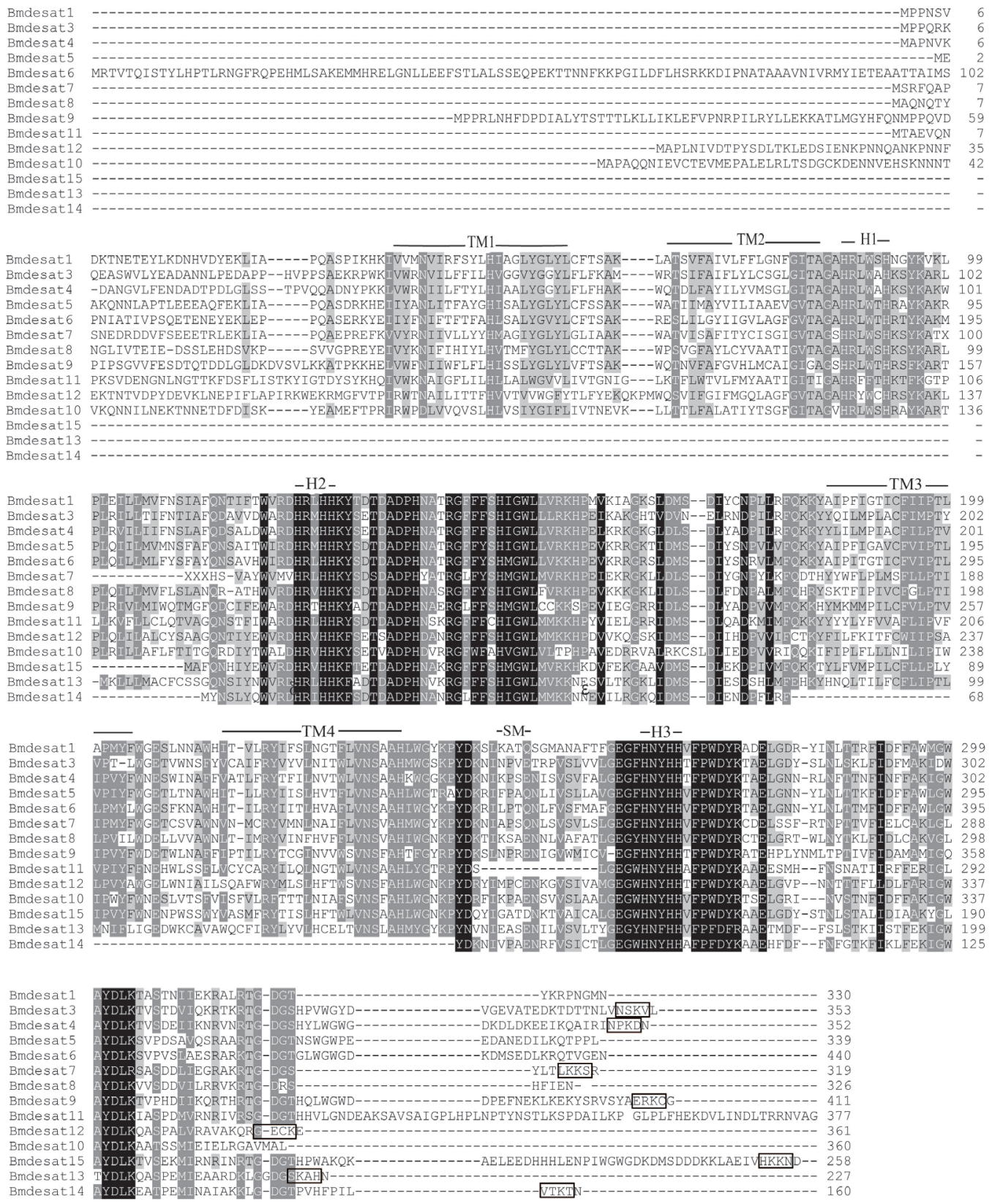

Figure 1. Aligned amino acid sequences encoded by silkworm putative desaturase genes. Black and grey backgrounds indicate amino acid identities and conservative substitutions, respectively. Three conserved histidine clusters of desaturases (H1-H3), the four transmembrane domains (TM1-TM4) and the signature motif (SM) are overlined. Boxed regions indicate the proposed ER retention signal. 
T. castaneum (Zhou et al., 2008). It is noteworthy that TcEFA12397, AgAGAP003050, and DmCG15531 formed a clade with a 99\% bootstrap value; their 3rd histidine motif differed from the conserved 3rd histidine domain (Figure S2), indicating fast evolution.

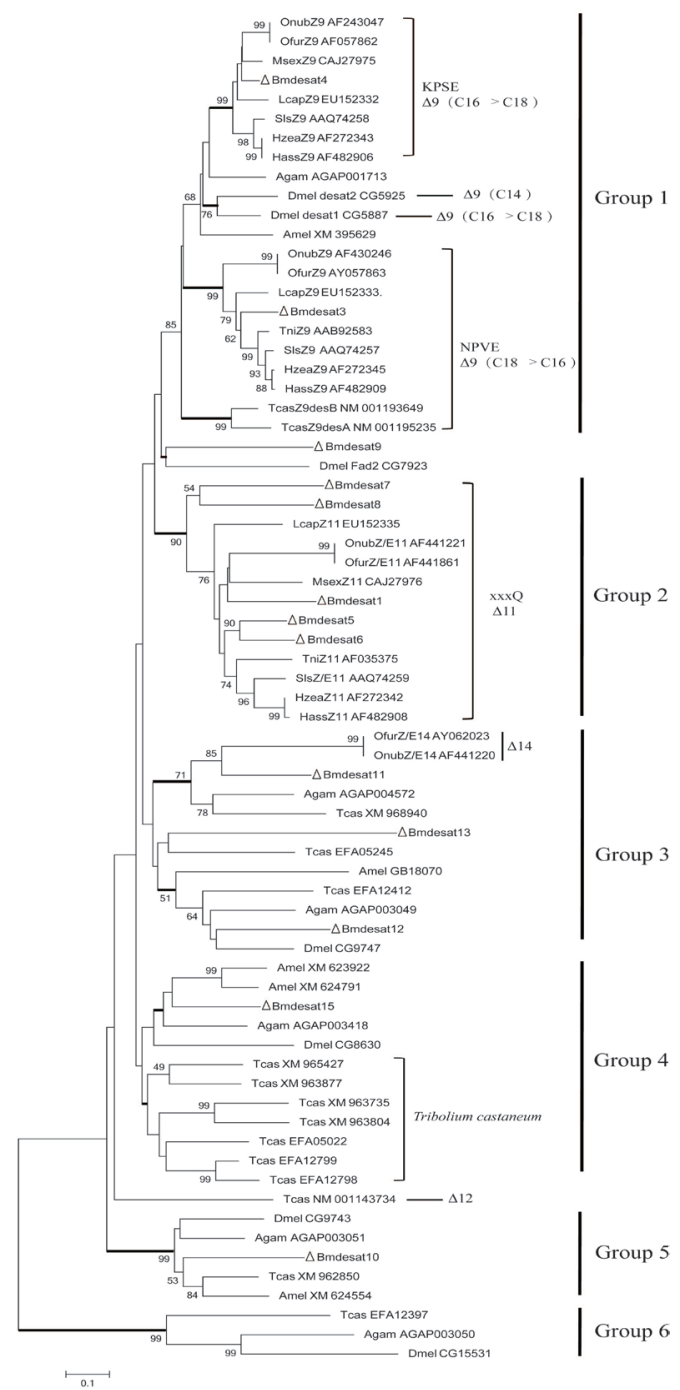

Figure 2. A neighbor-joining unrooted tree of fatty acid desaturase core domains (delimited by GAHR and EGFH motifs) among Bombyx mori, Drosophila melanogaster, Anopheles gambiae, Apis mellifera, Tribolium castaneum and other reported fatty acid desaturases, the result of multiple sequence alignment is showed in Figure S2. Numbers along branches indicate bootstrap support from 1000 replicates. The names for all sequences are composed of abbreviated species name and accession numbers $($ Dmel $=$ Drosophila melanogaster $:$ Agam $=$ Anopheles gambiae: Amel $=$ Apis mellifera : Tcas = Tribolium castaneum: Tni = Trichoplusia ni (Knipple et al., 1998, Liu et al., 1999); Onub/Ofur, Ostrinia species (Roelofs et al., 2002); Msex = Manduca sexta (Matouskova et al., 2007); Lcap = Lampronia capitella (Lienard et al., 2008); Sls = Spodoptera littoralis (Rodriguez et al., 2004, Serra et al., 2006); Hzea = Helicoverpa zea (Rosenfield et al., 2001); Hass = Helicoverpa assulta (Jeong et al., 2003), biochemical activities are indicated in connection to the species name. The desaturases from silkworm are indicated by a triangle. 


\section{Spatio-temporal expression profiles of silkworm fatty acid desaturase genes}

ESTs of silkworm fatty acid desaturase genes were identified. Twelve fatty acid desaturase genes matched at least $1 \mathrm{EST}$, and the coding regions of 4 fatty acid desaturase genes had complete ESTs. ESTs corresponding to Bmdesat1 and Bmdesat 5 were only found in the pheromone gland. ESTs for Bmdesat 12 and Bmdesat 15 were detected in follicle cells and the compound eye, respectively. ESTs for Bmdesat3, Bmdesat4, and Bmdesat 9 were from unfertilized embryos. Intriguingly, one EST for Bmdesat6 was present in the larval maxillary galea, which contains taste sensilla.

We further investigated the expression profiles of silkworm fatty acid desaturase genes in 9 different silkworm tissues on day 3 of the 5 th instar larvae based on microarray data (Xia et al., 2007). Bmdesat4 and Bmdesat10 were widely expressed in all surveyed tissues; they were particularly highly expressed in the midgut and Malpighian tubule (Figure 3). Bmdesat 13 was specifically detected in the anterior/median silk gland (A/MSG), meaning that it may have beneficial effects in sericin production. Bmdesat3, Bmdesat7, Bmdesat9, and Bmdesat15 were expressed in multiple tissues, such as head, testis, midgut, and Malpighian tubule. The expression patterns of these genes were similar in males and females except Bmdesat3. However, the other 7 fatty acid desaturase genes were not detected in any tissues on day 3 of the 5 th instar larvae.

We also profiled the expression of fatty acid desaturase genes during silkworm metamorphosis using microarray data (unpublished data) and semi-quantitative RT-PCR (Figure 4). Most fatty acid desaturase genes showed distinct expression patterns at 19 sequential

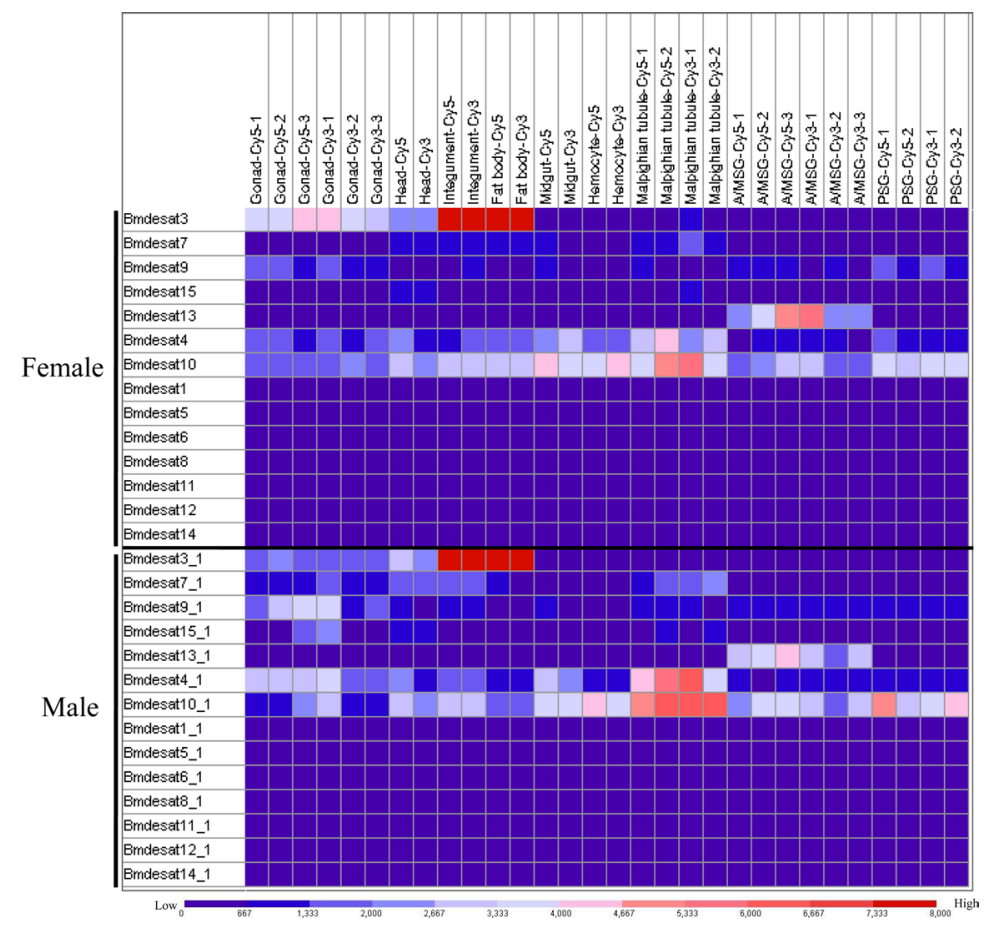

Figure 3. Expression profiles of desaturase genes in multiple larval tissues on day 3 of fifth instar of silkworm by microarray data. The expression levels are illustrated by red (higher expression) and blue (lower expression) boxes. $\mathrm{A} / \mathrm{MSG}=$ anterior/median silk gland; $\mathrm{PSG}=$ posterior silk gland $\mathrm{F}=$ female and $\mathrm{M}=$ male. 

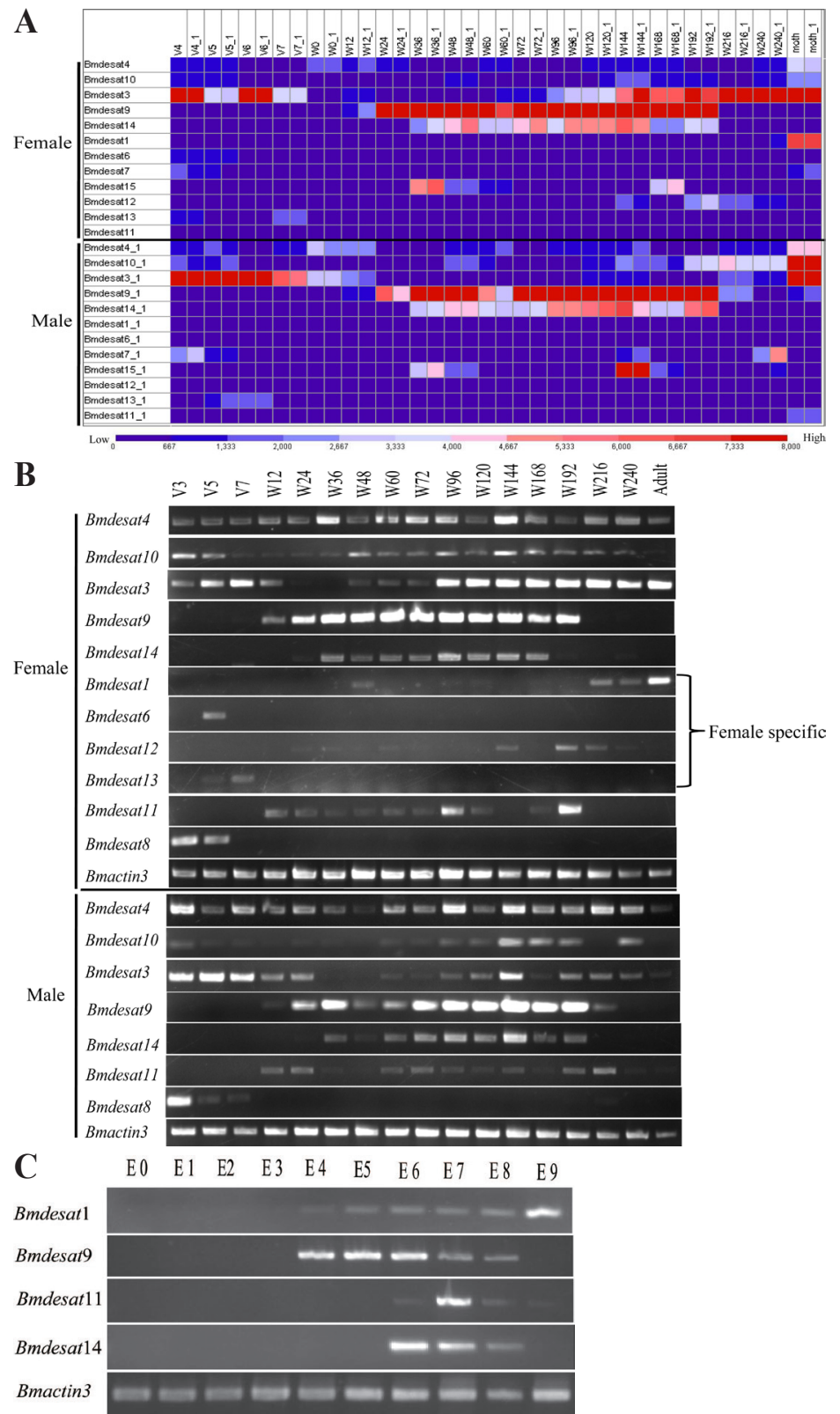

Figure 4. Expression profiles of fatty acid desaturase genes during silkworm metamorphosis based on microarray data (A) and RT-PCR (B, C). A. The expression levels are illustrated by red (higher expression) and blue (lower expression) boxes. The columns represents 19 different sample time points: V4 (day 4 of the fifth instar), V5, V6, V7, W0 (start of wandering), 13 different times after wandering (W12, W24, W36, W48, W60, W72, W96, W120, W144, W168, W192, W216, W240), and adult. B. Male and female individuals at 17 time points during silkworm metamorphosis (from V3 to moth) were selected for expression profiling using RT-PCR. The silkworm cytoplasmic actin A3 gene (GenBank accession No. U49854) was used as internal control, and denoted by Bmactin3. V means fifth larval instar; W means wandering. C. Expression profiling of embryonic development stages, E0-E9 means from day 0 to day 9 after oviposition. 
developmental time points from day 4 of the 5 th instar larvae to the adult (moth). Bmdesat1, Bmdesat6, and Bmdesat 12 were exclusively expressed at 2 or 3 developmental time points in females, and Bmdesat 1 was expressed in the late embryonic stage, suggesting that the gene may have a function other than bombykol production. Transcription levels of Bmdesat 3 were higher in females than in males. Bmdesat4 and Bmdesat10 were expressed almost over the entire metamorphosis, and Bmdesat8 was mainly detected in larvae. Bmdesat9, Bmdesat11, and Bmdesat 14 were expressed from spinning to the penultimate day before adult eclosion. Further investigation of their expression pattern during embryonic development stages revealed that they were expressed in the late embryo stages.

\section{DISCUSSION}

Using on genome dataset, we identified 14 fatty acid desaturase genes in silkworm. The deduced amino acid sequences of the fatty acid desaturase genes harbor 4 predicted transmembrane domains and 3 conserved histidine tracks, which are presumed to provide ligands to ferric iron in the catalytic center (Shanklin et al., 1994). Further analysis revealed that these motifs were localized at highly conserved positions of silkworm desaturases, and the location of the TM3 domain was well conserved in all of the fatty acid desaturase sequences of moths (Knipple et al., 2002). Although these genes have conserved functional domains and show high similarity, they have been clustered into different groups and display different expression profiles, indicating that they may have different functions.

\section{Genes may be involved in pupal oil production}

Silkworm pupae have a high nutritional value because of the presence of abundant fat, which constitutes about $30 \%$ of the total dry pupal weight; unsaturated fatty acid is abundant in pupal oil, reaching $66.8 \%$ of the total fat (Wei et al., 2009). The amount of the fatty acid in silkworm is far greater than that in mulberry leaves, indicating that many fatty acids in silkworm are the result of de novo synthesis in vivo. Polyunsaturated fatty acids were present in large amounts in silkworm eggs (Suzuki et al., 1970). Taken together, Bmdesat9, Bmdesat11, and Bmdesat14 were expressed during pupal development stages and the late embryonic development stages; thus, we presumed that they have a role in silkworm pupal oil production, and they supply abundant nutrition for pupal and embryonic development.

\section{Bmdesat3 may be involved in fatty acid metabolism}

Bmdesat 3 could be phylogenetically grouped within the clade of $\Delta 9(18 \mathrm{C}>16 \mathrm{C})$. It displayed high similarity $(>80 \%)$ to other moth $\Delta 9$ desaturases with a stearoyl-CoA preference, and it had an especially high similarity (86\%) to the $\Delta 9$ desaturase from Trichoplusia ni, which was a component of the metabolic $\Delta 9$ desaturase complex (Liu et al., 1999). Bmdesat 3 mRNA was enriched in the W72 stage (72 h after wandering; namely, $12 \mathrm{~h}$ after pupation) through the adult stage of females, a period when the ovum was grown, and it was also highly expressed in fat bodies on day 3 of 5 th instar larvae. Therefore, we suspected that Bmdesat 3 may be involved in fatty acid metabolism. 


\section{Bmdesat4 may be important for silkworm life}

Bmdesat4 is closely related to the other moth $\Delta 9$ desaturases with a 16-carbon substrate chain length preference, and it shared high sequence similarity to the fatty acid desaturase Dmdesat1 from D. melanogaster, which participates in Drosophila sex pheromone biosynthesis (Wicker-Thomas et al., 1997). However, in silkworm, Bmdesat1 was the only desaturase that was necessary to catalyze the 2 consecutive desaturation steps in sex pheromone biosynthesis (Moto et al., 2004). Therefore, Bmdesat4 may not be involved in the sex pheromone biosynthetic pathway. Interestingly, based on the genome resequencing of 40 domesticated and wild varieties, Bmdesat 4 was predicted to be a domestication-related candidate gene that was under artificial selection during domestication (Xia et al., 2009). Additionally, it was widely expressed in multiple tissues and constantly expressed from embryo to moth (Chen et al., 2012), suggesting that the gene may be important in silkworm, like housekeeping genes, but the concrete function of the gene is ambiguous.

\section{ACKNOWLEDGMENTS}

Research supported by grants from the National Hi-Tech Research and Development Program of China (\#2011AA100306), the National Basic Research Program of China (\#2012CB114600), and the National Natural Science Foundation of China (\#31172157).

\section{Supplementary material}

\section{REFERENCES}

Bai Y, Casola C, Feschotte C and Betran E (2007). Comparative genomics reveals a constant rate of origination and convergent acquisition of functional retrogenes in Drosophila. Genome Biol. 8: R11.

Chen QM, Cheng DJ, Ma ZG and Zhao P (2012). Cloning of full-length cDNA and promoter sequences of fatty acid desaturase gene desat 4 from silkworms, Bombyx mori and B. mandarina, and its prokaryotic expression. Acta Entomol. Sin. 55: 885-894.

Chertemps T, Duportets L, Labeur C, Ueyama M, et al. (2006). A female-specific desaturase gene responsible for diene hydrocarbon biosynthesis and courtship behaviour in Drosophila melanogaster. Insect Mol. Biol. 15: 465-473.

Dallerac R, Labeur C, Jallon JM, Knipple DC, et al. (2000). A delta 9 desaturase gene with a different substrate specificity is responsible for the cuticular diene hydrocarbon polymorphism in Drosophila melanogaster. Proc. Natl. Acad. Sci. U. S. A. 97: 9449-9454.

Goldsmith MR, Shimada T and Abe H (2005). The genetics and genomics of the silkworm, Bombyx mori. Annu. Rev. Entomol. 50: 71-100.

Hao G, Liu W, O'Connor M and Roelofs W (2002). Acyl-CoA Z9- and Z10-desaturase genes from a New Zealand leafroller moth species, Planotortrix octo. Insect Biochem. Mol. Biol. 32: 961-966.

Hastings N, Agaba M, Tocher DR, Leaver MJ, et al. (2001). A vertebrate fatty acid desaturase with Delta 5 and Delta 6 activities. Proc. Natl. Acad. Sci. U. S. A. 98: 14304-14309.

Holloway PW (1971). A requirement for three protein components in microsomal stearyl coenzyme A desaturation. Biochemistry 10: 1556-1560.

Horne I, Gibb N, Damcevski K, Glover K, et al. (2010). Two conserved Z9-octadecanoic acid desaturases in the red flour beetle, Tribolium castaneum. Gene 468: 41-47.

Jeong SE, Rosenfield CL, Marsella-Herrick P, Man YK, et al. (2003). Multiple acyl-CoA desaturase-encoding transcripts in pheromone glands of Helicoverpa assulta, the oriental tobacco budworm. Insect Biochem. Mol. Biol. 33: 609-622.

Knipple DC, Rosenfield CL, Miller SJ, Liu W, et al. (1998). Cloning and functional expression of a cDNA encoding a pheromone gland-specific acyl-CoA Delta11-desaturase of the cabbage looper moth, Trichoplusia ni. Proc. Natl. Acad. Sci. U. S. A. 95: 15287-15292. 
Knipple DC, Rosenfield CL, Nielsen R, You KM, et al. (2002). Evolution of the integral membrane desaturase gene family in moths and flies. Genetics 162: 1737-1752.

Li J, Ding SF, Habib NA, Fermor BF, et al. (1994). Partial characterization of a cDNA for human stearoyl-CoA desaturase and changes in its mRNA expression in some normal and malignant tissues. Int. J. Cancer 57: 348-352.

Li Y, Monroig O, Zhang L, Wang S, et al. (2010). Vertebrate fatty acyl desaturase with Delta4 activity. Proc. Natl. Acad. Sci. U. S. A. 107: 16840-16845.

Lineard MA, Stranth M, Hedenstrom E, Johansson T, et al. (2008). Key biosynthetic gene subfamily recruited for pheromone production prior to the extensive radiation of Lepidoptera. BMC Evol. Biol. 8: 270.

Liu W, Ma PW, Marsella-Herrick P, Rosenfield CL, et al. (1999). Cloning and functional expression of a cDNA encoding a metabolic acyl-CoA delta 9-desaturase of the cabbage looper moth, Trichoplusia ni. Insect Biochem. Mol. Biol. 29: 435-443.

Los DA and Murata N (1998). Structure and expression of fatty acid desaturases. Biochim. Biophys. Acta 1394: 3-15.

Matouskova P, Pichova I and Svatos A (2007). Functional characterization of a desaturase from the tobacco hornworm moth (Manduca sexta) with bifunctional Z11- and 10,12-desaturase activity. Insect Biochem. Mol. Biol. 37: 601-610.

Matsumoto S, Hull JJ, Ohnishi A, Moto K, et al. (2007). Molecular mechanisms underlying sex pheromone production in the silkmoth, Bombyx mori: characterization of the molecular components involved in bombykol biosynthesis. $J$. Insect Physiol. 53: 752-759.

Matsumoto S (2010). Molecular mechanisms underlying sex pheromone production in moths. Biosci. Biotechnol. Biochem. 74: 223-231.

Moto K, Suzuki MG, Hull JJ, Kurata R, et al. (2004). Involvement of a bifunctional fatty-acyl desaturase in the biosynthesis of the silkmoth, Bombyx mori, sex pheromone. Proc. Natl. Acad. Sci. U. S. A. 101: 8631-8636.

Murata $\mathrm{N}$ and Wada H (1995). Acyl-lipid desaturases and their importance in the tolerance and acclimatization to cold of cyanobacteria. Biochem. J. 308: 1-8.

Rodríguez S, Hao G, Liu W, Pina B, et al. (2004). Expression and evolution of delta9 and delta11 desaturase genes in the moth Spodoptera littoralis. Insect Biochem. Mol. Biol. 34: 1315-1328.

Roelofs WL, Liu W, Hao G, Jiao H, et al. (2002). Evolution of moth sex pheromones via ancestral genes. Proc. Natl. Acad. Sci. U.S. A. 99: 13621-13626.

Rogers MJ and Strittmatter P (1973). Lipid-protein interactions in the reconstitution of the microsomal reduced nicotinamide adenine dinucleotide-cytochrome b 5 reductase system. J. Biol. Chem. 248: 800-806.

Rosenfield CL, You KM, Marsella-Herrick P, Roelofs WL, et al. (2001). Structural and functional conservation and divergence among acyl-CoA desaturases of two noctuid species, the corn earworm, Helicoverpa zea, and the cabbage looper, Trichoplusia ni. Insect Biochem. Mol. Biol. 31: 949-964.

Serra M, Pina B, Bujons J, Camps F, et al. (2006). Biosynthesis of 10,12-dienoic fatty acids by a bifunctional Delta11 desaturase in Spodoptera littoralis. Insect Biochem. Mol. Biol. 36: 634-641.

Shanklin J, Whittle E and Fox BG (1994). Eight histidine residues are catalytically essential in a membrane-associated iron enzyme, stearoyl-CoA desaturase, and are conserved in alkane hydroxylase and xylene monooxygenase. Biochemistry 33: 12787-12794.

Shanklin J and Cahoon EB (1998). Desaturation and related modifications of fatty acids1. Annu. Rev. Plant Physiol. Plant Mol. Biol. 49: 611-641.

Spatz L and Strittmatter P (1971). A form of cytochrome b5 that contains an additional hydrophobic sequence of 40 amino acid residues. Proc. Natl. Acad. Sci. U. S. A. 68: 1042-1046.

Strittmatter P, Spatz L, Corcoran D, Rogers MJ, et al. (1974). Purification and properties of rat liver microsomal stearyl coenzyme A desaturase. Proc. Natl. Acad. Sci. U. S. A. 71: 4565-4569.

Stukey JE, McDonough VM and Martin CE (1990). The OLE1 gene of Saccharomyces cerevisiae encodes the delta 9 fatty acid desaturase and can be functionally replaced by the rat stearoyl-CoA desaturase gene. J. Biol. Chem. 265: 20144-20149.

Suzuki M, Kobayashi M and Kekawa NI (1970). Variation of triglycerides and fatty acid methyl esters in silkworm eggs during embryonic development. Lipids 5: 539-544.

Thiede MA, Ozols J and Strittmatter P (1986). Construction and sequence of cDNA for rat liver stearyl coenzyme A desaturase. J. Biol. Chem. 261: 13230-13235.

Wei ZJ, Liao AM, Zhang HX, Liu J, et al. (2009). Optimization of supercritical carbon dioxide extraction of silkworm pupal oil applying the response surface methodology. Bioresour. Technol. 100: 4214-4219.

Wicker-Thomas C, Henriet C and Dallerac R (1997). Partial characterization of a fatty acid desaturase gene in Drosophila melanogaster. Insect Biochem. Mol. Biol. 27: 963-972.

Xia Q, Cheng D, Duan J, Wang G, et al. (2007). Microarray-based gene expression profiles in multiple tissues of the domesticated silkworm, Bombyx mori. Genome Biol. 8: R162. 
Xia Q, Guo Y, Zhang Z, Li D, et al. (2009). Complete resequencing of 40 genomes reveals domestication events and genes in silkworm (Bombyx). Science 326: 433-436.

Yoshiga T, Okano K, Mita K, Shimada T, et al. (2000). cDNA cloning of acyl-CoA desaturase homologs in the silkworm, Bombyx mori. Gene 246: 339-345.

Zhou XR, Horne I, Damcevski K, Haritos V, et al. (2008). Isolation and functional characterization of two independentlyevolved fatty acid Delta12-desaturase genes from insects. Insect Mol. Biol. 17: 667-676. 